\title{
QUEUEING SYSTEM WITH PASSIVE SERVERS
}

\author{
ALEXANDER N. DUDIN and VALENTINA I. KLIMENOK \\ Byelorussian State University \\ Department of Applied Mathematics \\ Minsk, Belarus
}

(Received January, 1995; Revised January, 1996)

\begin{abstract}
In this paper the authors introduce systems in which customers are served by one active server and a group of passive servers. The calculation of response time for such systems is rendered by analyzing a special kind of queueing system in a synchronized random environment. For an embedded Markov chain, sufficient conditions for the existence of a stationary distribution are proved. A formula for the corresponding vector generating function is obtained. It is a matrix ana$\log$ of the Pollaczek-Khinchin formula and is simultaneously a matrix functional equation. A method for solving this equation is proposed.
\end{abstract}

Key words: Queueing System, Synchronized Random Environment (SRE), Two-Dimensional Embedded Markov Chain, Linear Matrix Functional Equation.

AMS (MOS) subject classifications: 60K25, 68M20, 90B22.

\section{Introduction}

The participation of several service devices (servers) when processing customers is a typical feature of real-world processes which occur in computer networks, databases and other systems. Situations including the parallel operation of servers are described by multichannel queueing systems, and they have been investigated in detail. Successive service corresponds to multiphase queues; their extensions are subject to queueing networks. In some systems that have been studied to a far lesser extent, it is impossible to represent the processing of customers as a sequence of complete operations in terms of known physical or logical devices. We shall describe one such system below.

The service of customers is performed by one active server and $W$ identical passive servers. An active server processes customers itself and, moreover, it supervises and schedules a necessary number of passive servers. Each passive server is assigned to a customer by the active server. So, it is possible to have a waiting line of customers with a few idle passive servers. This explains the term "passive server".

Examples of such situations are as follows. Let us consider the transmission of data packets at the transport level of a LAN (Local Area Network) using the window protocol. This process is supervised by the processor of a transport station. The processor is an active server. The window protocol (methods of slipping windows or credits) permits the transmission of at most $W$ packets without receiving a confirmation (receipt) of a successful transmission (see e.g., [22]). Practically, the control is performed as follows. There are $W$ teller-timers (passive servers). After transmitting the packet at a channel level of the LAN, the group of timers must be turned on. The 
group size corresponds to the number of station-receivers of this package. If there is a lack of turned-off timers, the active server pauses or breaks off the transmission of the packets (depending on a realization of the transport protocol). Every timer turns off after either the confirmation is received or the time-out of the confirmation is finished. The service of a packet by the processor is performed in two stages. The first stage consists of reading the packet and searching turned-off timers. The second stage consists of the generation of package transmission at a channel level.

It will be seen that the mathematical model presented in Section 2 corresponds to the above process. An applied research project of a LAN transport station performance motivated our investigation of queueing systems with passive servers.

Another example is as follows. Consider the processing SQL-operators, which require the establishing of exclusive locks imposed on pages of a relational database table. An active server is the database manager system (see e.g., [3]). Passive servers are the table pages on a magnetic-disk storage device.

Consequently, queueing systems with passive servers are adequate mathematical models for important real-world systems. However, they are neither described nor studied in the scientific literature. That is why the present investigation of such systems is both novel and of interest.

This paper is devoted to the evaluation of customer response time in such systems. Section 2 contains a formal description of the customer service process. It turns out that in spite of a close similarity with queueing systems, this model cannot be described in terms of any classical queueing system. However, it may be successfully described as a queueing system in a Synchronous Random Environment (SRE). The corresponding model is formulated in Section 3. The rest of the paper is concerned with calculation of response time.

\section{Description of the Basic Service System}

Customers arrive at the system according to a stationary Poisson process with rate $\lambda$. The service of customers is performed by one active server and $W$ passive servers. At first, a customer is served by an active server. If this server is busy upon customer's arrival, the customer lines up. The waiting room is of infinite capacity. Customers are picked from the queue according to the FIFO discipline. The service of a customer by an active server consists of two phases. The durations of the $r$ th phase are independent, identically distributed random variables with the DF $B_{r}(x)$, the Laplace-Stieltjes transform $\beta_{r}(s)=\int_{0}^{\infty} \exp (-s x) d B_{r}(x)$, and finite moments $b_{k}^{(r)}=$ $\int_{0}^{\infty} x^{k} d B_{r}(x), k=1,2, r=1,2$.

Upon the completion of the first phase, the passive servers are engaged in service. Let $\xi$ be the number of passive servers required for the service of a given customer. Here $\xi$ is an integervalued random variable distributed as $q_{m}=P\{\xi=m\}$, where $q_{m}>0, m=\overline{0, W}, \sum_{m=0}^{W} q_{m}=1$.

Some passive servers can become idle upon completion of the first phase by the active server. At this moment, other passive servers may be processing customers arrived earlier. If the number of free passive servers is not less than the number of servers required to process a given customer (this number is defined as the realization of the random variable $\xi$ ), the passive servers begin to process this customer. At the same time, the second phase of the service of this customer by the active server begins. If the available passive servers are insufficient, there are two possible modes or variants of the system's behavior. The first one (variant \#1): the active server interrupts the service of a customer. The customer leaves the system unprocessed. Variant \#2: the active server waits until a necessary number of passive servers become free. After that, the second phase of service by the active server begins. A passive server, switched to a busy state by the 
active server, remains in this state during a random time having an exponential distribution with parameter $\gamma$. The passive server transfers into a free state without interference from an active server after this random time expires. We shall also distinguish two different ways of employing passive servers. For variant $\boldsymbol{a}$, the period of passive service begins upon the completion of the second stage of service by the active server, while for variant $\boldsymbol{b}$ it begins at the start of the second stage of service by the active server.

A combination of variants 1,2 and $\boldsymbol{a}, \boldsymbol{b}$ gives four cases 1.a, 1.b, 2.a, and 2.b.

By the sojourn time we mean the total time spent by a customer in the system (from its arrival until departure). Throughout this paper, we will target the mean sojourn time (called also response time) of customers. However, our results can be used for finding higher order moments and the Laplace-Stieltjes transform of the sojourn time distribution.

The way we described the service process in a basic system seems to enable us the use of traditional queueing theory methods for investigating this process. But, unfortunately we run into considerable difficulties when trying to present the basic system in terms of any known classical queueing system. Among other complications, it is very difficult to identify the concept of a "server", moreover to set a joint distribution of service times of successive customers. At the same time, the processing of the basic system may be successfully described in terms of a queueing system in a SRE. By a queueing system in a random environment, we mean a queueing system with input intensity and/or system distribution dependent upon the state of some external random process (so-called random environment). This kind of system has attracted the attention of queueing theorists (see, for example S. Asmussen [1], A. Dudin [4, 6-8], G. Falin [9], B. Gnedenko and I. Kovalenko [12], L. Goel [14], S. Halfin [15], M. van Hoorn and L. Seelen [16], Ya. Kogan and V. Litvin [19], I. Korotaev [20, 21], P. Naor and U. Yechiali [33], M. Neuts [23], C. O'Cinneide and P. Purdue [24], N. Prabhu and Y. Zhu [25], P. Purdue [26], G. Regterschot and J. de Smit [27], T. Rolski [28], J. Sztrik [31], H. Takahashi and H. Akimaru [32]). Queueing systems in SRE are those in a random environment for which state transactions are synchronized with those of the queue. Corresponding systems were considered in $[5,11,18,29,30]$.

In the following section, we shall describe the basic system in terms of a queueing system in SRE.

\section{The Queueing Systems in SRE}

We establish the following correspondence between the terms of the above basic system and the queueing system in SRE.

\section{Basic System}

an active server

the number of busy passive

servers at a given moment

\section{Queueing System in SRE}

a server

the state of the synchronous random environment at a given moment

Taking in account these analogs, we will interpret the basic system in terms of a queueing system in SRE as follows.

Customers arrive singly according to a Poisson process with rate $\lambda$ and line up. There is unlimited waiting space. The queue discipline is first-come-first-served. The service time of each customer consists of two phases. The durations of the $r$ th phase are independent, identically dis- 
tributed variables with common distribution function $B_{r}(x), r=1,2$. There is a random environment - process $\eta_{t}$ - the number of busy passive servers in the basic system at the moment $t$ - with state space $\{0,1, \ldots, W\}$. The queueing system and the random environment interact in the following way. The random variable $\xi$ is activated upon completion of the first phase of service. $\xi$ is associated with the number of passive servers needed to process a given customer, and it has distribution $q_{m}=P\{\xi=m\}, m=\overline{0, W}$. A realization of $\xi$ is added to the current value of $\eta_{t}$. If the sum exceeds $W$, the customer either leaves the system without the second phase of service (variant \#1) or waits until the value of $\xi+\eta_{t}$ drops to $W$ and then it immediately begins the second phase of service (variant \#2). Otherwise (if the sum does not exceed the value $W$ ) the second phase starts immediately. The state of the random environment increases by the value of $\xi$ either upon completion (variant $\boldsymbol{a}$ ) or at the moment of starting (variant $\boldsymbol{b}$ ) the second phase of service.

Between two consecutive moments of jumps of $\eta_{t}$, the process $\eta_{t}$ behaves as a pure death process with state space $\{0,1, \ldots, W\}$ and death rate $\gamma$.

It is easily seen that this queueing system in SRE describes adequately the queueing process in the basic system. It is also easy to see that the total sojourn time of a customer in the basic system equals the sojourn time in the queueing system in SRE and the time between the completion of service by an active server and the moment of freeing all passive channels servicing the given customer. Consequently, the expected sojourn time (response time) of a customer in the basic system is the sum of the expected sojourn time (response time) of a customer in the queueing system in SRE and the expected value of the time till the passive servers are released.

Let us begin the calculation of customer's response time in the queueing system in SRE.

We will use the following notation:

- $t_{k}$ is the departure moment of the $k$ th customer from the queueing system in SRE (the moment when an active server of the basic system completes the service of the $k$ th customer);

- $i_{t_{k}}$ is the number of customers in the system at the moment $t_{k}+0$ (the length of queue in the basic system at the moment $t_{k}-0$ );

- $\eta_{t_{k}}$ is the state of random environment at the moment $t_{k}+0$ (the number of busy passive servers in the basic system at the moment $\left.t_{k}+0\right)$;

- $P\{(i, v) \rightarrow(l, r)\}=P\left\{i_{t_{k+1}}=l, \eta_{t_{k+1}}=r \mid i_{t_{k}}=i, \eta_{t_{k}}=v\right\}$ are transition probabilities of the Markov chain $\left\{i_{t_{k}}, \eta_{t_{k}}\right\}, k \geq 1$;

- $R_{v, r}(z)=\sum_{l=i-1}^{\infty} z^{l-i+1} P\{(i, v) \rightarrow(l, r)\},|z| \leq 1$ is the generating function of the corresponding transition probabilities, $v, r=\overline{0, W}$;

ties; $\quad R(z)=\left(R_{v, r}(z)\right)_{v}=\overline{0, W}, r=\overline{0, W}$ is the matrix generating function of transition probabili-

- $\delta_{v, r}(t)=C_{v}^{r} e^{-r \gamma t}\left(1-e^{-\gamma t}\right)^{v-r}, 0 \leq r \leq v$, is the transition probability of the death process with rate $\gamma$ from state $v$ to state $r$ during a period of time of length $t, \delta_{v, r}(t)=0$, if $r>v$;

- $\psi(j, v, r, n)=\int_{0}^{\infty} \frac{(\lambda t)^{j}}{j !} e^{-\lambda t} \delta_{v, r}(t) d B_{n}(t), j \geq 0,0 \leq r \leq v \leq W, n=1,2$ is the probability of the arrival of $j$ customers from the Poisson stationary input flow with rate $\lambda$ and the transition of the death process with rate $\gamma$ from state $v$ to state $r$ during the time having the distribution function $B_{n}(t)$;

$$
\text { - } \quad \varphi_{n}(z, v, r)=\sum_{j=0}^{\infty} \psi(j, v, r, n) z^{j}=\int_{0}^{\infty} e^{-\lambda t(1-z)} \delta_{v, r}(t) d B_{n}(t), \quad 0 \leq r \leq v \leq W, \quad|z| \leq 1,
$$

$n=1,2$ is the generating function of probabilities $\psi(j, v, r, n)$; 
- $F_{v, r}(t)$ is the distribution function of transition time of the death process with rate $\gamma$ from state $v$ to state $r, 0 \leq r \leq v \leq W$;

$F_{v, r}(t) ; \quad f_{v, r}(s)=\int_{0}^{\infty} e^{-s t} d F_{v, r}(t)=\prod_{n=r+1}^{v} \frac{n \gamma}{n \gamma+s}$ are Laplace-Stieltjes transforms of functions - $\nabla_{v, r}=\int_{0}^{\infty} \lambda e^{-\lambda t} \delta_{v, r}(t) d t$ is the probability of the transition of the death process with rate $\gamma$ from state $v$ to state $r$ during the interval between successive arrivals of customers from the stationary Poisson flow with rate $\lambda$;

- $\nabla=\left\|\nabla_{v, r}\right\|_{v=\overline{0, W}, r=\overline{0, W}}$

- $\pi(i, v)=\lim _{k \rightarrow \infty} P\left\{i_{t_{k}}=i, \eta_{t_{k}}=v\right\}, i \geq 0, v=\overline{0, W}$,

is the stationary distribution of the Markov chain $\left\{i_{t_{k}}, \eta_{t_{k}}\right\}, k \geq 1$;

- $\Pi_{v}(z)=\sum_{i=0}^{\infty} \pi(i, v) z^{i},|z| \leq 1$ is the generating function of the probabilities $\pi(i, v)$;

- $\left.\vec{\Pi}(z)=\stackrel{i=0}{\left(\Pi_{0}\right.}(z), \Pi_{1}(z), \ldots, \Pi_{W}(z)\right)^{T}$;

- $T$ stands for vector-matrix transpose.

- $E$ is $(W+1) \times(W+1)$ identity matrix.

\section{Stationary Distribution of Markov Chain $\left\{i_{t_{k}}, \eta_{t_{k}}\right\}$}

Let us solve the problem of finding the stationary distribution $\pi(i, v), i \geq 0, v=\overline{0, W}$.

First of all, we shall specify conditions for the existence of this distribution.

Theorem 1: A sufficient condition of existence of limits (1) is the validity of the inequality

$$
\left.(-1)^{W} D^{\prime}(z)\right|_{z=1}<0
$$

where

$$
D(z)=\operatorname{det}[R(z)-z E]
$$

A proof is given in Appendix A.

Now we establish the main result that allows us to find the distribution $\pi(i, v), i \geq 0$, $v=\overline{0, W}$.

Theorem 2: The vector $\vec{\Pi}(z)$ of generating functions $\Pi_{v}(z), v=\overline{0, W}$, satisfies the following formula:

$$
\vec{\Pi}(z)=\left(R^{T}(z)-z E\right)^{-1} R^{T}(z)\left(E-z \nabla^{T}\right) \vec{\Pi}(0) .
$$

Expression (4) may be called the matrix Pollaczek-Khinchin formula. It is a matrix analog of the well-known formula for the generating function of the queue length distribution for $M / G / 1$ system.

Proof: Stationary probabilities $\pi(i, v)$ of the embedded Markov chain $\left\{i_{t_{k}}, \eta_{t_{k}}\right\}, k \geq 0$, are determined from the system of linear algebraic equations:

$$
\pi(l, r)=\sum_{v=0}^{W} \sum_{i=0}^{l+1} \pi(i, v) P\{(i, v) \rightarrow(l, r)\}, l \geq 0 .
$$


Multiply corresponding equations (5) by $z^{l}$ and sum up the equations in $l$ from 0 to $\infty$. Transform the obtained expression, taking into account that the transition probabilities $P\{(i, v) \rightarrow$ $(l, r)\}$ depend on $l-i$ and do not depend on $l$ and $i$ separately. (Note that such a property of transition probabilities makes sense for the above notation for $R_{v, r}(z)$.) While transforming, we use the evident relation

$$
P\{(0, v) \rightarrow(l, r)\}=\sum_{m=0}^{v} \nabla_{v, m} P\{(1, m) \rightarrow(l, r)\} .
$$

In the end, we obtain the following system of linear algebraic equations for generating functions $\Pi_{r}(z)$ :

$$
z \Pi_{r}(z)=\sum_{v=0}^{W} \Pi_{v}(z) R_{v, r}(z)+\sum_{v=0}^{W} \pi(0, v)\left[z \sum_{m=0}^{v} \nabla_{v, m} R_{m, r}(z)-R_{v, r}(z)\right], r=\overline{0, W}
$$

or in the matrix form

$$
\left(R^{T}(z)-z E\right) \vec{\Pi}(z)=R^{T}(z)\left(E-z \nabla^{T}\right) \vec{\Pi}(0)
$$

Equation (7) implies (4) in an obvious way.

Remark: For the case of our system, we have established the matrix Pollaczek-Khinchin formula for the generating function $\vec{\Pi}(z)$. In fact, it can be shown that the same result is valid for any two-dimensional Markov chain when one component of the chain is denumerable, the other one is finite and transition probabilities depend only on the difference between the values of denumerable components. A proof of this more general result is going to be published separately.

To apply formula (4) we need expressions for the elements $R_{v, r}(z)$ of matrix $R(z)$. To obtain these expressions, we analyze the behavior of the queue and the environment during the interval of time between successive departures. Using the formula for composite probability, we obtain the explicit form of the transition probabilities $P\{(i, v) \rightarrow(l, r)\}$. Multiplying these probabilities by $z^{l}$ and summing them up, we prove the following result. follows:

Theorem 3: Generating functions $R_{\boldsymbol{v}, r}(z)$ in the above variants $1 . \boldsymbol{a}, \mathbf{1 . b}, \mathbf{2 . a}, \mathbf{2 . b}$ are defined as

1. Variant 1.a:

$$
R_{v, r}(z)=\sum_{k=0}^{v} \varphi_{1}(z, v, k) \sum_{m=\max \{0, r-k\}}^{W-k} q_{m} \varphi_{2}(z, k+m, r)+\underset{m=}{\varphi_{1}(z, v, r) \sum_{m-r+1}^{W}} q_{m} .
$$

2. Variant 1.b:

$$
R_{v, r}(z)=\sum_{k=0}^{v} \varphi_{1}(z, v, k) \sum_{m=\max \{0, r-k\}}^{\min \{W-k, r\}} q_{m} \varphi_{2}(z, k, r-m)+\varphi_{1}(z, v, r) \sum_{m=W-r+1}^{W} q_{m} .
$$

3. Variant 2.a:

$$
\begin{aligned}
& R_{v, r}(z)=\sum_{k=0}^{v} \varphi_{1}(z, v, k) \\
& \times \quad\left\{\sum_{m=\max \{0, r-k\}}^{W-k} q_{m} \varphi_{2}(z, k+m, r)+\varphi_{2}\left(\begin{array}{c}
\left.W, W, r) \sum_{m=W+1-k}^{W} q_{m} f_{k, W-m}(\lambda(1-z))\right\} .
\end{array}\right.\right.
\end{aligned}
$$

4. Variant $2 . b$ : 


$$
\begin{aligned}
& R_{v, r}(z)=\sum_{k=0}^{v} \varphi_{1}(z, v, k) \\
& \times\left\{\sum_{m=\max \{0, r-k\}}^{\min \{W-k, r\}} q_{m} \varphi_{2}(z, k, r-m)+\sum_{m=W-k+1}^{r} q_{m} \varphi_{2}(z, W-m, r-m) f_{k, W-m}(\lambda(1-z))\right\} .
\end{aligned}
$$

$0 \leq r \leq v \leq W$

Formulas (8)-(11) allow us to obtain an explicit expression of the matrix $R(z)$ utilized in (4).

The vector $\vec{\Pi}(z)$, defined by (4), has to comply with the normalization condition:

$$
\vec{\Pi}^{T}(1) \mathbf{1}=1
$$

where $\mathbf{1}=(1, \ldots, 1)^{T}$.

For the classical M/G/1 system (the system without auxiliary passive servers), the scalar ana$\log$ of formula (4) and the normalization condition (12) define completely the value of the generating function of the queue length distribution. Unfortunately, our case is more complicated. Expression (4) gives the value of the generating function $\vec{\Pi}(z)$ for all $|z| \leq 1$, except for $z=0$. But this value is unknown. Essentially, equation (4) is the linear matrix functional equation in vector-function $\vec{\Pi}(z)$.

A similar equation,

$$
A(s) \vec{\Phi}(s)=C(s) \vec{\Phi}(+\infty)
$$

occurs in investigations of queueing systems in the asynchronous Markov random environment (see e.g., $[4,9,12,15])$. Here $\vec{\Phi}(s)$ is a vector-function. Its components are Laplace-Stieltjes transforms of the distribution functions of queue length under different current values of the environment. The matrices $A(s)$ and $C(s)$ are known.

A standard way of solving (13) is as follows (see e.g., $[4,9,12,15]$ ). Let us introduce $M(s)=A^{-1}(s) \operatorname{det} A(s)$. Form a system of linear algebraic equations for components of vector $\vec{\Phi}(+\infty)$ using the analyticity of the vector $\vec{\Phi}(s)$ on the right half of the complex plane and take one equation from every system

$$
M\left(s_{k}\right) C\left(s_{k}\right) \vec{\Phi}(+\infty)=0, \quad k=\overline{0, W} .
$$

Here $s_{0}=0$ is a root of the equation $\operatorname{det} A(s)=0, s_{k}$ are roots of this equation in the right half-plane, $k=\overline{1, W}$. The case of multiple roots can be taken into account separately. Next we use the additional fact:

$$
\vec{\Phi}(0)=P,
$$

where $P$ is the stationary distribution vector of the environment. $P$ is known a priori. Eliminate the uncertainty of $M(s) C(s) \vec{\Phi}(+\infty) / \operatorname{det} A(s)$ at the point $s=0$ and obtain an inhomogeneous algebraic equation for components of vector $\vec{\Phi}(+\infty)$, using (15). We include this equation instead of any homogeneous equation in the above formed system. Then we solve this equation having a nonsingular matrix. As the result, we have a known vector $\vec{\Phi}(+\infty)$. This way, functional equation (13) is transformed into a linear matrix equation. Such an equation is solved by using standard techniques.

We can apply the same idea to solve the linear matrix functional equation (7). That is why 
we will not discuss the solution in detail. We shall note only some key points.

In spite of the fact that problems of solving (7) and (13) are similar, the problem of solving (7) is more complicated. An additional difficulty is connected with the lack of any additional information (except the normalization condition) concerning the value of the vector $\vec{\Pi}(z)$ at any fixed point, in particular at the point $z=1$. Therefore we have to make additional efforts to find components of the vector $\vec{\Pi}(0)$ in comparison with the above plan. We will outline the additional plan briefly.

Form the homogeneous system of linear algebraic equations for components of vector $\vec{\Pi}(0)$ as it was done above for the components of vector $\vec{\Phi}(\infty)$. In this connection we will make use of the validity of the following assertion.

Theorem 4: The determinant $D(z)=\operatorname{det}\left(R^{T}(z)-z E\right)$ has exactly $W$ roots in the region $\{|z|<1\}$ and one simple root at $z=1$.

The proof of this theorem is given in Appendix B.

We will perform the following preliminary elementary operations to form an inhomogeneous equation for the components of vector $\vec{\Pi}(0)$.

Sum up the equations of system (6) to obtain the following relation:

$$
\sum_{v=0}^{W} \Pi_{v}(z)\left(\sum_{r=0}^{W} R_{v, r}(z)-z\right)=\sum_{v=0}^{W} \pi(0, v)\left(\sum_{r=0}^{W} R_{v, r}(z)-z \sum_{m=0}^{v} \nabla_{v, m} \sum_{r=0}^{W} R_{m, r}(z)\right) .
$$

Expanding both sides of (16) in Taylor series at $z=1$ and equating corresponding coefficients, we obtain the following system of relations:

$$
\sum_{v=0}^{W}\left(\Pi_{v}^{(l)}(1) \psi_{v}^{(0)} \frac{(-1)^{l}}{l !}+\sum_{i=1}^{l-1} \Pi_{v}^{(i)}(1) \psi_{v}^{(l-i)} \frac{(-1)^{i}}{i !}\right)=\sum_{v=0}^{W} \pi(0, v) \Xi_{v}^{(l)}, l \geq 0 .
$$

Here $\Pi_{v}^{(l)}(1)$ is the value of the $l$ th derivative of function $\Pi_{v}(z)$ at $z=1$. An explicit expression for coefficients $\psi_{v}^{(l)}, \Xi_{v}^{(l)}, l \geq 0$ is given in Appendix C.

Consider system (7) at $z=1$. As it follows from Theorem 4, the matrix $R^{T}(1)-E$ is singular. Replace one equation of system (7) by equation (17) for $l=0$. We can show that the matrix of the modified system is nonsingular. Inverting this matrix and multiplying it by the right-hand side of the modified system, we practically determine the relation $\vec{\Pi}(1)=S \vec{\Pi}(0)$. Here $S$ is the known matrix. Using the normalization condition (12) we obtain the equation

$$
\vec{\Pi}^{T}(0) S^{T} 1=1 .
$$

Equation (18) is a desired inhomogeneous equation. Including this equation in the above formed system for the components of the vector $\vec{\Pi}(0)$ we solve this system and obtain the value of vector $\vec{\Pi}(0)$. This way, functional equation (7) is reduced to a simple linear matrix equation. It is easy to solve. To find the components of the vector $\vec{\Pi}(z)$ or its derivatives at the point $z=1$ we can use equation (7) or its derivatives at $z=1$. To obtain a nonsingular matrix of the corresponding system we have to replace one equation of this system by the equation of system (17) for an appropriate value of $l$.

A more detailed version of the above algorithm is not included in the text of this paper and will be published separately. 


\section{The Calculation of Customer's Response Time}

As mentioned above, the response time of a customer in the basic system equals the total length of two time periods. The first one, $V_{A}$, is the time from customer's arrival till his departure from an active server (that is, a response time in the above described queueing system in SRE). The second one, $V_{p}$, is the time from the departure of a customer from an active server until the moment when all passive servers involved in service of the given customer are released.

At first we consider the calculation of the value $V_{A}$. Taking into account properties of the stationary Poisson flow, it is not difficult to see that the following analog of Little's formula is valid:

$$
V_{A}=\lambda^{-1} \sum_{v=0}^{W} \Pi_{v}^{\prime}(1)
$$

Using (19) and values of $\Pi_{v}^{\prime}(1), v=\overline{0, W}$, derived by our algorithm, it is easy to calculate the value of $V_{A}$.

The process of releasing passive servers involved in service of the given customer is the death process. It makes the calculation of the value $V_{p}$ not very difficult. The result is different for each variant of the system's behavior.

1. Variant 1.a:

$$
V_{p}=\sum_{m=1}^{W} q_{m} \sum_{v=0}^{W} \Phi_{v} \sum_{r=0}^{W-m} \int_{0}^{\infty} \delta_{v, r}(t) d B_{1}(t) \sum_{l=1}^{m} \frac{\gamma^{-1}}{l}
$$

2. Variant 1.b:

$$
V_{p}=\sum_{m=1}^{W} q_{m} \sum_{v=0}^{W} \Phi_{v} \sum_{r=0}^{-m} \int_{0}^{\infty} \delta_{v, r}(t) d B_{1}(t) \sum_{i=0}^{m} \int_{0}^{\infty} \delta_{m, i}(t) d B_{2}(t) \sum_{l=1}^{i} \frac{\gamma^{-1}}{l}
$$

where $\Phi_{v}$ is the probability that $v$ passive servers are busy at the starting moment of service of an arbitrary customer by an active server:

$$
\Phi_{v}=\Pi_{v}(1)-\pi(0, v)+\sum_{m=v}^{W} \pi(0, m) \nabla_{m, v}
$$

3. Variant 2.a:

$$
V_{p}=\sum_{m=1}^{W} q_{m} \sum_{l=1}^{m} \frac{\gamma^{-1}}{l}
$$

4. Variant 2.b:

$$
V_{p}=\sum_{m=1}^{W} q_{m} \sum_{r=0}^{m} \int_{0}^{\infty} \delta_{m, r}(t) d B_{2}(t) \sum_{l=1}^{r} \frac{\gamma^{-1}}{l} .
$$

By the way, we adduce formulas for calculating the value $P_{\text {reject }}$. Here $P_{\text {reject }}$ is the probability that a customer leaves the system without the second phase of service by the active server. This characteristic is essential for variants $1 . a$ and $1 . b$ :

$$
P_{\text {reject }}=\sum_{m=1}^{W} q_{m} \sum_{v=W+1-m}^{W} \Phi_{v} \sum_{r=W+1-m}^{v} \int_{0}^{\infty} \delta_{v, r}(t) d B_{1}(t) .
$$




\section{Conclusion}

A mathematical model of a customer's service by one active and a random number of passive devices was investigated in this paper. Such a model describes adequately a wide collection of operating technical and physical devices and processes. The problem of the response time calculation for such systems was solved by the reduction of the basic model to the queueing system in the special kind of SRE. An approach of finding the stationary distribution was developed. This approach was realized in an algorithm. The algorithm is coded as a C++ program for IBM-compatible personal computers. This program was used for the computation of characteristics of several real systems and its efficiency was verified. The employment of the approach and the algorithm makes it possible to solve problems of performance evaluation, capacity planning and the optimization of system characteristics such as: the optimal choice of the value $W$ (receive and send windows width, multiprogramming level and so on), the value $\gamma^{-1}$ (the time-out of the transmitting protocol, performance of passive servers), the throughput of a system and so on.

\section{References}

[1] Asmussen, S., Ladder heights and the Markov-modulated M/G/1 queue, Stochastic Processes and Their Applications 37 (1991), 313-326.

[2] Bellman, R., Introduction of Matrix Analysis, McGraw-Hill, New York 1960.

[3] Date, C., A Guide to DB2, Addison-Wesley Publishing Company, Reading, MA 1984.

[4] Dudin, A., Analysis of characteristics of data transmission process in an ISDN channel, Automatic Control and Computer Sciences, Allerton Press, New York 6 (1987), 42-49.

[5] Dudin, A., About queueing system $\mathrm{M} / \mathrm{G} / 1$ in cyclic synchronous random environment, XIV All-Union Workshop in Computer Networks 3 (1989), 44-48.

[6] Dudin, A., Computation of the characteristics of an $M / G / 1$ queueing system operating in the memoryless semi-Markovian random environment, Technique of Telecommunications 7 (1990), 30-38.

[7] Dudin, A. and Markov, A., Computation of the characteristics of a queueing system operating in the cyclic semi-Markovian random environment, XV All-Union Workshop in Computer Networks 2 (1990), 241-246.

[8] Dudin, A., The algorithm of computation of the characteristics of an M/G/1 queueing system in the semi-Markovian random environment, Fourth International Seminar in Teletraffic Theory and Computer Simulation, GPNTB, Moscow (1992), 51-55.

[9] Falin, G., About single server queueing system with varying service rate, Eng. Cybernetics (Izvestia of the Soviet Acad. Sci. ) 1 (1988), 134-137.

[10] Gantmakher, F., The Theory of Matrices, Science, Moscow 1967.

[11] Gelenbe, E. and Rosenberg, C., Queues with slowly varying arrival and service processes, Management Sciences 36:8 (1990), 928-937.

[12] Gnedenko, B. and Kovalenko, I., Introduction to Queueing Theory, Science, Moscow 1966.

[13] Gnedenko, B., Danieljan, E., Dimitrov, B., Klimov, G., and Matveev, V., Priority Queues, Moscow University, Moscow 1973.

[14] Goel, L., Transient solution of a certain type of heterogeneous queues, Trabajos de Estadistica y de Investigacion Operativa 30:3 (1979), 63-70.

[15] Halfin, S., The backlog of data in buffers with variable input and output rates, Performance of Computer-Communication Systems, Elsevier Science Publishers BV, North-Holland (1984), 307-319.

[16] van Hoorn, M. and Seelen, L., The SPP/G/1 queue: a single server queue with a switched Poisson process as input process, O.R. Spektrum 5:4 (1983), 207-218. 
[17] Khomichkov, I., About stationary regime existence in local area network with 1-persistent protocol, XVIII All-Union Workshop in Computer Networks (1992), 61-65.

[18] Klimenok, V., Characteristics computation of queueing system M/G/1 in synchronous random environment, Analysis and Synthesis of Queueing Systems and Computer Networks, Odessa 1 (1990), 87-92.

[19] Kogan, Ya. and Litvin, V., Piecewise diffusion approximations for queueing problems with heterogeneous arrivals and service, Problems of Control and Information Theory 8:5-6 (1979), 433-443.

[20] Korotaev, I., Queueing Systems with Varying Parameters, Tomsk University 1991.

[21] Korotaev, I. and Spivak, R., Queueing systems operating in the semi-Markovian random environment, Automation and Remote Control 7 (1992), 86-92.

[22] Kuo, F., Protocols and Techniques for Data Communication Networks, Prentice-Hall, New Jersey 1981.

[23] Neuts, M., Matrix-Geometric Solutions in Stochastic Models, Johns Hopkins University Press, Baltimore and London 1981.

[24] O'Cinneide, C. and Purdue, P., The M/M/ $\infty$ queue in a random environment, Journal of Applied Probability 23:1 (1986), 175-184.

[25] Prabhu, N. and Zhu, Y., Markov modulated queueing systems, Queueing Systems 5 (1989), 215-246.

[26] Purdue, P., The M/M/1 queue in a Markovian environment, Operations Research 22:3 (1974), 562-569.

[27] Regterschot, G. and de Smit, J., The queue M/G/1 with Markov modulated arrivals and services, Mathematics of Operations Research 11:3 (1986), 465-483.

[28] Rolski, T., Upper bounds of single server queues with doubly stochastic Poisson arrivals, Mathematics of Operations Research 11:3 (1986), 442-450.

[29] Sotelo, W., Mukumoto, K. and Fukuda, a., On multiserver queues with $m$-phase synchronous fluctuation of traffic intensity, Trans. of the IEICE E70:12 (1987), 1197-1194.

[30] Sotelo, W., Mukumoto, K. and Fukuda, A., Some properties of queueing systems with fluctuating traffic intensity, Trans. of the IEICE E71 (1988), 659-668.

[31] Sztrik, J., On the heterogeneous M/G/n blocking system in a random environment, J. of the Operational Research Society 38:1 (1987), 57-63.

[32] Takahashi, H. and Akimaru, H., A diffusion model for queues in randomly varying environment, Trans. of the IEICE 1 (1986), 13-20.

[33] Yechiali, U. and Naor, P., Queueing problems with heterogeneous arrivals and service, Oper. Res. 19 (1971), 722-734. 


\section{Appendix A}

Proof of Theorem 1: We will apply Moustafa's theorem [13]. The statement below is an ana$\log$ of this theorem for our case.

Sufficient conditions for the Markov chain $\left\{i_{t_{k}}, v_{t_{k}}\right\} k \geq 1$, to have a stationary distribution are formulated as follows:

- the chain is irreducible and aperiodic;

- there are $\varepsilon>0$, a positive integer $j_{0}$, and a set of nonnegative numbers $x_{i, v}, i \geq 0, v=\overline{0, W}$, such that the following inequalities are valid

$$
\begin{gathered}
\sum_{i=0}^{\infty} \sum_{r=0}^{W} P\{(j, v) \rightarrow(i, r)\} x_{i, r}-x_{j, v}<-\varepsilon, j>j_{0}, v=\overline{0, W} \\
\sum_{i=0}^{\infty} \sum_{r=0}^{W} P\{(j, v) \rightarrow(i, r)\} x_{i, r}<\infty, j \leq j_{0}, \quad v=\overline{0, W}
\end{gathered}
$$

To ensure the irreducibility and aperiodicity of the chain, it is sufficient to write down expressions for the transition probabilities of the Markov chain.

Let

$$
x_{i, r}=i+1+\beta_{r}, \quad i \geq 0, \quad r=\overline{0, W}
$$

where $\beta_{r}, r=\overline{0, W}$, are some real numbers.

Demonstrate that there is a set of numbers $x_{i, r}, i \geq 0, r=\overline{0, W}$, having the form (A.3) for which the conditions of Moustafa's theorem are valid.

Substituting (A.3) into (A.1), we obtain the system of inequalities

$$
\sum_{r=0}^{W} R_{v, r}^{\prime}(1)-1+\sum_{r=0}^{W} \beta_{r} R_{v, r}(1)-\beta_{v}<-\varepsilon, v=\overline{0, W} .
$$

Let's verify that under condition (2) of Theorem 1 there are $\varepsilon>0$ and numbers $\beta_{r}, r=\overline{0, W}$, such that inequalities (A.4) hold.

Consider the system consisting of $W$ equations and one inequality

$$
\begin{gathered}
\sum_{r=0}^{W} R_{v, r}^{\prime}(1)-1+\sum_{r=0}^{W} \beta_{r} R_{v, r}(1)-\beta_{v}=0, v=\overline{W-1} \\
\sum_{r=0}^{W} R_{W, r}^{\prime}(1)-1+\sum_{r=0}^{W} \beta_{r} R_{W, r}(1)-\beta_{W}<0 .
\end{gathered}
$$

(The idea of the transition from (A.4) to (A.5), (A.6) was derived from [17]). Let us interpret relations (A.5) as the system of linear algebraic equations for $\beta_{0}, \beta_{1}, \ldots, \beta_{W-1}$. Rewrite (A.5) in the form

$$
\begin{gathered}
\sum_{r=0}^{v-1} R_{v, r}(1) \beta_{r}+\left(R_{v, v}(1)-1\right) \beta_{v}+\sum_{r=v+1}^{W-1} R_{v, r}(1) \beta_{r}=1-\sum_{r=0}^{W} R_{v, r}^{\prime}(1)-\beta_{W} R_{v, W}(1), \\
v=\overline{0, W-1} .
\end{gathered}
$$


Taking into account the nature of $R_{v, r}(z)$, it is easy to see that the coefficients in (A.7) satisfy Hadamard's conditions:

$$
\left|R_{v, v}(1)-1\right|>\sum_{\substack{r=0 \\ r \neq v}}^{W-1} R_{v, r}(1), v=\overline{0, W-1}
$$

Therefore, the determinant $d$ of (A.7) is not equal to zero [10]. Then (A.7) has the unique solution

$$
\beta_{v}=\frac{d_{v}}{d}, v=\overline{0, W-1}
$$

where $d_{v}$ is the determinant obtained from $d$ by replacing the $v$ th column with the column of constant terms in (A.7).

It is easy to see that system (A.7) considered as the system of linear algebraic equations for $\beta_{0}, \beta_{1}, \ldots, \beta_{W-1}, \beta_{W}$ has an infinite set of solutions. Each of them satisfies (A.8) and is the solution of the basic system (A.5). Find the condition under which an arbitrary solution of (A.5) will satisfy inequality (A.6).

Expanding determinants $d_{v}, v=\overline{0, W-1}$, in the entries of the $v$ th column and substituting the obtained expression for $\beta_{v}, v=\overline{0, W-1}$, into (A.6), we get the following inequality

$$
\begin{gathered}
-\frac{\beta_{W}}{d}\left\{\left[R_{W, W}(1)-1\right] d-\sum_{v=0}^{W-1} R_{W, v}(1) \sum_{k=0}^{W-1} R_{k, W}(1) \Delta_{k, v}\right\} \\
+\frac{1}{d}\left\{-\left[1-\sum_{r=0}^{W} R_{W, r}^{\prime}(1)\right] d+\sum_{v=0}^{W-1} R_{W, v}(1) \sum_{k=0}^{W-1}\left[1-\sum_{r=0}^{W} R_{k, r}^{\prime}(1)\right] \Delta_{k, v}\right\}<0 .
\end{gathered}
$$

Here $\Delta_{k, v}$ is the cofactor of the entry located in the intersection of the $k$ th row and the $v$ th column of determinant $d_{v}$.

It is easy to prove that the expression in the first braces in (A.9) is $D(1)$. To see this, it is sufficient to observe that the sum $-\sum_{k=0}^{W-1} R_{k, W}(1) \Delta_{k, v}$ is the cofactor of the entry indexed by $W$ and $v$ in the determinant $D(1), v=\overline{0, W-1}$. Similarly, the expression in the second braces in (A.9) is the determinant obtained from $D(1)$ by replacing the $W$ th column with the column

$$
\left(1-\sum_{r=0}^{W} R_{0, r}^{\prime}(1), 1-\sum_{r=0}^{W} R_{1, r}^{\prime}(1), \ldots, 1-\sum_{r=0}^{W} R_{W, r}^{\prime}(1)\right)^{T} \text {. }
$$

It is easy to see that the obtained determinant is the derivative of $D(z)$ at point $z=1$.

Then, inequality (A.9) may be rewritten in the form

$$
-\frac{\beta_{W}}{d} D(1)+\frac{1}{d} D^{\prime}(1)<0
$$

or, taking into account that $D(1)=0$ (see the proof of Theorem 4), we have

$$
\frac{D^{\prime}(1)}{d}<0
$$

The sign of the determinant $d$ is the same as the sign of $(-1)^{W}$. To prove this fact, we will write $d$ in the form

$$
d=\operatorname{det}\left\|R^{(W-1)}(1)-E^{(W-1)}\right\|
$$


where $R^{(W-1)}(1)$ is the matrix $\left\|R_{v, r}(1)\right\|_{v=\overline{0, W-1}}$ and $E^{(W-1)}$ is the $W \times W$ identity matrix. $r=\overline{0, W-1}$

The entries of the matrix $R^{(W-1)}(1)$ satisfy the conditions

$$
R_{v, r}(1) \geq 0, v, r=\overline{0, W-1}, \sum_{r=0}^{W-1} R_{v, r}(1)<1, v=\overline{0, W-1} .
$$

It allows us to classify the matrix as the special Minkowski-Leontjev's matrix. For such class of matrices it is proved in [2] that

$$
\operatorname{det}\left\|E^{(W-1)}-R^{(W-1)^{T}}(1)\right\|>0
$$

(A.11) may be rewritten in the form

$$
d=(-1)^{W} \operatorname{det}\left\|E^{(W-1)}-R^{(W-1)^{T}}(1)\right\|
$$

The last relation and inequality (A.12) prove the above statement about the sign of the determinant $d$. Since sign $d=\operatorname{sign}(-1)^{W},($ A.10) is the equivalent to inequality (2).

Thus, under condition (2) of Theorem 1, system (A.5)-(A.6) has an infinite set of solutions. Let $\beta^{0}=\left(\beta_{0}^{0}, \beta_{1}^{0}, \ldots, \beta_{W}^{0}\right)$ be an arbitrary solution of system (A.5)-(A.6). This solution is a point of the $(W+1)$-dimensional space, located on the line formed by the intersection of hyperplanes (A.5). In addition, this point belongs to the $(W+1)$-dimensional half-space defined by inequality (A.6). It is not difficult to see that at a neighborhood of point $\beta^{0}$, we can find a point $\beta^{*}$ with coordinates $\beta_{0}^{*}, \beta_{1}^{*}, \ldots, \beta_{W}^{*}$, so that for some number $\varepsilon_{v}>0$, the following inequalities are valid:

$$
\sum_{r=0}^{W} R_{v, r}^{\prime}(1)-1+\sum_{r=0}^{W} \beta_{r}^{*} R_{v, r}(1)-\beta_{v}^{*}<-\varepsilon_{v}, \quad v=\overline{0, W} .
$$

Let $\varepsilon=\max _{0 \leq v \leq W} \varepsilon_{v}$. In view of (A.13) for such $\varepsilon$ and $\beta^{*}$ inequalities (A.4) hold true. Then for our $\varepsilon$ and $x_{i, r}^{*}=i+1+\beta_{r}^{*}, i \geq 0, r=\overline{0, W}$, inequalities (A.1) are valid for any $j$. But under the condition of Moustafa's theorem, numbers $x_{i, r}^{*}$ should be nonnegative. Generally speaking, this condition is not satisfied in our case because arbitrarily large negative numbers in modulus may occur among the numbers $\beta_{r}^{*}, r=\overline{0, W}$. But taking into account that inequalities (A.1) should be correct only for $j>j_{0}$ we can take the required set of nonnegative numbers $x_{i, r}, i \geq 0, r=\overline{0, W}$, in the form

where $j_{0}>\max _{0 \leq r \leq W}\left|\beta_{r}^{*}\right|$.

$$
x_{i, r}=\left\{\begin{array}{cc}
0, & i<j_{0} \\
x_{i, r}^{*}, & i \geq j_{0}
\end{array}\right.
$$

In such a way, we have demonstrated that there are positive $\varepsilon$ and a set of nonnegative numbers $x_{i, r}, i \geq 0, r=\overline{0, W}$, such that for any $r, 0 \leq r \leq W$, and $j>j_{0}$ inequalities (A.1) are valid. It is easy to see that for the same $x_{i, r}, i \geq 0, r=\overline{\overline{0}}, \overline{\bar{W}}$, and $j_{0}$, inequalities (A.2) are correct also.

\section{Appendix B}

Proof of Theorem 4: First introduce some notations and prove some lemmas.

Denote 


$$
D^{(n)}(z)=\operatorname{det}\left(R^{(n)}(z)-E^{(n)} z\right), \quad n=\overline{0, W}
$$

where the matrix $R^{(n)}(z)$ is derived from the matrix $R(z)$ by deleting last $W-n$ rows and columns; $E^{(n)}$ is the identity $(n+1) \times(n+1)$ matrix.

The correspondence between notations is as follows:

$$
R^{(W)}(z) \equiv R(z), E^{(W)} \equiv E, D^{(W)}(z) \equiv D(z)
$$

Introduce also

$$
D_{\nu}^{(n)}(z)=\operatorname{det}\left(R_{v}^{(n)}(z)-E^{(n)} z\right), n=\overline{0, W-1},
$$

where the matrix $R_{v}^{(n)}(z)$ is derived from matrix $R^{(n)}(z)$ by replacing the $v$ th column with the part $\left(R_{0, n+1}(z), R_{1, n+1}(z), \ldots, R_{n, n+1}(z)\right)^{T}$ of the column of matrix $R(z)$.

Besides that, introduce the region of the plane:

$$
\begin{gathered}
S_{\varepsilon}=\{|z|<1,|z-1|<\varepsilon\}, \\
S_{\varepsilon}^{(1)}=S_{\varepsilon} \cap\{\operatorname{Re} z \leq 1\}, S_{\varepsilon}^{(2)}=S_{\varepsilon} \cap\{\operatorname{Re} z>1], \varepsilon>0 .
\end{gathered}
$$

Denote by $\gamma_{S_{\varepsilon}}$ the boundary of the region $S_{\varepsilon}$, by $\gamma_{S_{\varepsilon}^{(1)}}$ a part of the curve $\gamma_{S_{\varepsilon}}$ in the region $\operatorname{Re} z \leq 1$ and by $\gamma_{S_{\varepsilon}(2)}$ a part of the curve $\gamma_{S_{\varepsilon}}$ in the region $\operatorname{Re} z>1$. ly.

Let $\gamma_{S_{\varepsilon}^{(1)}}, \gamma_{S_{\varepsilon}(2)}$ be parts of the curve $\gamma_{S_{\varepsilon}}$ belonging to the regions $\operatorname{Re} z \leq 1, \operatorname{Re} z>1$ respective-

Lemma B.1: Any function $R_{v, r}(z), v, r=\overline{0, W}$, complies with the inequality

$$
\left|R_{v, r}(z)\right|_{\gamma_{\varepsilon}^{(1)}} \leq R_{v, r}(1)
$$

Proof: Using the definition of the function $\varphi_{n}(z, v, r)$ it is not difficult to demonstrate the validity of the inequalities:

$$
\left|\varphi_{n}(z, v, r)\right|_{\operatorname{Re} z \leq 1} \leq \varphi_{n}(1, v, r), \quad v, r=\overline{0, W}, \quad n=1,2 .
$$

Using (7)-(10) and (B.2), it is not difficult to prove that

$$
\left|R_{v, r}(z)\right|_{\operatorname{Re} z \leq 1} \leq R_{v, r}(1) .
$$

But because the curve $\gamma_{S}(1)$ completely belongs to the region $\operatorname{Re} z \leq 1$, the inequality (B.1) follows immediately from (B.3).

Lemma B.2: The following inequalities hold:

$$
\left|R_{v, v}(z)-z\right|_{\gamma_{\varepsilon}^{(1)}}>\sum_{\substack{i \neq 0 \\ i \neq v}}^{W-1}\left|R_{v, i}(z)\right|_{\gamma_{\varepsilon}(1)}, v=\overline{0, W-1},
$$




$$
\left|R_{W, W}(z)-z\right|_{\gamma_{\varepsilon}(1)} \geq \sum_{i=0}^{W-1}\left|R_{W, i}(z)\right|_{\gamma_{\varepsilon}(1)}
$$

Proof: Using Lemma B.1 and properties of generating functions $R_{v, r}(z)$ we have the following sequence of relations:

$$
\begin{gathered}
\left|R_{v, v}(z)-z\right|_{S_{\varepsilon}^{(1)}} \geq|z|_{\gamma_{\varepsilon}^{(1)}}-\left|R_{v, v}(z)\right|_{\gamma_{\varepsilon}^{(1)}} \geq 1-R_{v, v}(1)=\sum_{\substack{i=0 \\
i \neq j}}^{W} R_{v, i}(1) \\
v=\overline{0, W}
\end{gathered}
$$

Recalling that, due to the nature of the system, $R_{\nu, W}(1)>0$ and taking into account the result of Lemma B.1, we obtain:

$$
\begin{gathered}
\sum_{\substack{i \neq 0 \\
i \neq v}}^{W} R_{v, i}(1)>\sum_{\substack{i \neq 0 \\
i \neq v}}^{W-1}\left|R_{v, i}(z)\right|_{\gamma_{\varepsilon}^{(1)}}, v=\overline{0, W-1} \\
\sum_{i=0}^{W-1} R_{W, i}(1) \geq \sum_{i=0}^{W-1}\left|R_{W, i}(z)\right|_{\gamma_{\varepsilon}^{(1)}}
\end{gathered}
$$

Inequalities (B.4), (B.5) follow from (B.6), (B.7).

Lemma B.3: The following inequalities hold:

$$
\left|D^{(n)}(z)\right|_{\gamma_{\varepsilon}^{(1)}}>\left|D_{v}^{(n)}(z)\right|_{\gamma_{\varepsilon}^{(1)}}, \quad v=\overline{0, n}, n=\overline{0, W-2} .
$$

Proof: For $n=0$, the validity of Lemma B.3 follows, from inequality (B.4). Let now, $n>0$. Prove the correctness of (B.8) for some $z_{0} \in \gamma_{S_{\varepsilon}^{(1)}}$. Consider the following system of linear alge-
braic equations:

$$
\left(R^{(n)}\left(z_{0}\right)-z_{0} E^{(n)}\right) x=\left(R_{0, n+1}\left(z_{0}\right), R_{1, n+1}\left(z_{0}\right), \ldots, R_{n, n+1}\left(z_{0}\right)\right)^{T}
$$

In view of (B.4), the entries of matrix $R^{(n)}\left(z_{0}\right)$ satisfy Hadamard's conditions [10]. It implies that this matrix is nonsingular and system (B.9) has a unique solution $x^{0}=\left(x_{0}^{0}, x_{1}^{0}, \ldots, x_{n}^{0}\right)$ in the complex plane. Demonstrate that the entries of vector $x^{0}$ satisfy the inequalities

$$
\left|x_{i}^{0}\right|<1, i=\overline{0, n}
$$

Suppose the contrary; namely, that

$$
\max _{0 \leq i \leq n}\left|x_{i}^{0}\right|=\left|x_{v}^{0}\right| \geq 1 .
$$

Consider the $v$ th equation of system (B.9) rewritten in the form

$R_{v, v}\left(z_{0}\right)-z_{0}=-\sum_{\substack{i=0 \\ i \neq v}}^{n} R_{v, i}\left(z_{0}\right) \frac{x_{i}^{0}}{x_{v}^{0}}+R_{v, n+1}\left(z_{0}\right) \frac{1}{x_{v}^{0}}$

Because of our assumption $\left|\frac{x_{i}^{0}}{\frac{x_{v}^{0}}{x_{v}}}\right| \leq 1,\left|\frac{1}{i \neq v} x_{v}^{0}\right| \leq 1$, we obtain from (B.11) the following inequality: 
This contradicts Hadamard's conditions.

$$
\left|R_{v, v}\left(z_{0}\right)-z_{0}\right| \leq \sum_{\substack{i \neq 0 \\ i \neq v}}^{n+1}\left|R_{v, i}\left(z_{0}\right)\right|
$$

Thus entries of a vector satisfying (B.9) satisfy (B.10) too. But according to the Cramer's rule

$$
\left|x_{i}^{0}\right|=\frac{\left|D_{v}^{(n)}\left(z_{0}\right)\right|}{\left|D^{(n)}\left(z_{0}\right)\right|}, \quad i=\overline{0, n} .
$$

It implies the validity of (B.8) at any point $z_{0}$ of the curve $\gamma_{S^{(1)}}$.

Corollary B.1: Assertions of Lemma B.1-Lemma B.3 are valid at any point of contour $C=$ $\{|z|=1\}$.

Proof: The correctness of this statement for $z=0$ is established by the same way as it was done for all other points $z \in \gamma_{S_{\varepsilon}(1)}, \varepsilon \rightarrow 0$, of contour $C$.

Lemma B.4: Every function $R_{v, v}(z)-z, \quad v=\overline{0, W}$ has exactly one zero in the region $|z|<1$.

Proof: The validity of (B.1) for any point of contour $C$ and inequalities $R_{v, v}(1)<1$, $v=\overline{0, W}$, imply that

or

$$
\begin{gathered}
\left|R_{v, v}(z)\right|_{C}<1, v=\overline{0, W} \\
\left|R_{v, v}(z)\right|_{C}<|z|_{C} .
\end{gathered}
$$

The last inequality and Rouche's theorem yields the proof of Lemma B.4.

Lemma B.5: The determinant $D^{(n)}(z), \quad n=\overline{0, W-1}$, has exactly $n+1$ zeros in the region $|z|<1$.

Proof (by induction in $n$ ):

1. $n=0$. Proof follows from Lemma B.4.

2. $1 \leq n \leq W-1$. Let the determinant $D^{(n-1)}(z)$ have exactly $n$ zeros in the region $|z|<1$. We prove that $D^{(n)}(z)$ has exactly $n+1$ zeros in the same region by showing the validity of the inequality

$$
\left|D^{(n)}(z)-\left(R_{n, n}(z)-z\right) D^{(n-1)}(z)\right|_{C}<\left|\left(R_{n, n}(z)-z\right) D^{(n-1)}(z)\right|_{C} .
$$

Decomposing $D^{(n)}(z)$ in entries of the last row and dividing inequality (B.12) by its non-zero right-hand side we obtain the equivalent inequality:

$$
\left|\sum_{i=0}^{n-1} \frac{R_{n, i}(z)}{\left(R_{n, n}(z)-z\right)} \frac{\Delta_{n, i}^{(n)}(z)}{D^{(n-1)}(z)}\right|_{C}<1 .
$$

Here $\Delta_{n, i}^{(n)}(z)$ is the cofactor of the entry located at the $n$th row and the $i$ th column of the determinant $D^{(n)}(z)$. The validity of Lemmas 2-3 at all points of contour $C$ implies the validity of the inequalities:

$$
\frac{\sum_{i=0}^{n-1}\left|R_{n, i}(z)\right|_{C}}{\left|R_{n, n}(z)-z\right|_{C}}<1, \frac{\left|\Delta_{n, i}^{(n)}(z)\right|_{C}}{\left|D^{(n-1)}(z)\right|_{C}}<1, \quad i=\overline{0, n}, \quad n=\overline{0, W-1} .
$$


These inequalities prove (B.13) and (B.12).

Inequalities (B.12) and Rouche's theorem imply that in the region $|z|<1$ the function $D^{(n)}(z)$ has the same number of zeros as the function $\left(R_{n, n}(z)-z\right) D^{(n-1)}(z)$. But from Lemma B.4 and the suggestion of the induction, it follows that the last function has exactly $n+1$ zeros in the region $|z|<1$.

Corollary B.2: The function $\left(R_{W, w}(z)-z\right) D^{(W-1)}(z)$ has exactly $W+1$ zeros in the region $S_{\varepsilon}, \varepsilon \rightarrow 0$.

Proof: It is easy to see that when $\varepsilon \rightarrow 0$, the region $S_{\varepsilon}=\{z:|z|<1\} \cup\{1\}$. Lemma B.4 and Lemma B.5 imply that the function under consideration has exactly $W+1$ zeros in the region $|z|<1$. It is not difficult to demonstrate that the point $z=1$ is not a zero of this function. Therefore, all zeros of this function in the region $S_{\varepsilon}, \varepsilon \rightarrow 0$, are concentrated in the region $|z|<1$ and there are $W+1$ such zeros.

Proof of Theorem 4: The point $z=1$ is a root of the function $D^{(W)}(z)$ because the determinant $D^{(W)}(1)$ can be reduced to a determinant that has the zero column. Hence, the assertion of Theorem 4 is equivalent to the assertion that the function $D^{(W)}(z)$ has exactly $W+1$ zeros in the region $S_{\varepsilon}, \varepsilon \rightarrow 0$ (see the reasoning about the structure of this region in the proof of the Corollary B.2). Let us prove this modified assertion.

Since the functions $D^{(W)}(z),\left(R_{W, W}(z)-z\right) D^{(W-1)}(z)$ are formed as a result of summation and multiplication of generating functions, these functions are analytic in the region $S_{\varepsilon}, \varepsilon \rightarrow 0$.

We prove that these functions satisfy the following inequality on the boundary $\gamma_{S_{\varepsilon}}$ of the region $S_{\varepsilon}, \varepsilon \rightarrow 0$ :

$$
\left|D^{(W)}(z)-\left(R_{W, W}(z)-z\right) D^{(W-1)}(z)\right|_{\gamma_{S_{\varepsilon}}}<\left|\left(R_{W, W}(z)-z\right) D^{(W-1)}(z)\right|_{\gamma_{S_{\varepsilon}}} .
$$

The inequality (B.14) is equivalent to the inequalities

$$
\begin{gathered}
\left|\frac{D^{(W)}(z)}{\left(R_{W, W}(z)-z\right) D^{(W-1)}(z)}-1\right|<1, \\
\left|\frac{D^{(W)}(z)}{\left(R_{W, W}(z)-z\right) D^{(W-1)}(z)}-1\right|_{\gamma_{\varepsilon}^{(2)}}^{2}<1 .
\end{gathered}
$$

Decomposing the determinant $D^{(W)}(z)$ in the entries of the $W$ th row and using the results of Lemma B.2 and Lemma B.3, which is valid for $n=W-1$ too, it is not difficult to verify (B.15).

We now prove (B.16). Expand the function that is minuend on the left-hand side of (B.16) in Taylor series at point $z=1$. Taking into account the relation $D^{(W)}(1)=0$, we obtain:

$$
\frac{D^{(W)}(z)}{\left(R_{W, W}(z)-z\right) D^{(W-1)}(z)}=\frac{D^{\prime(W)}(1)}{\left(R_{W, W}(1)-1\right) D^{(W-1)}(1)}(z-1)+o(|z-1|) .
$$

Using (B.17) and the representation

$$
z=1+\varepsilon(\cos \varphi+i \sin \varphi),-\frac{\pi}{2}<\varphi<\frac{\pi}{2}
$$

on the curve $\gamma_{S_{\varepsilon}^{(2)}}$ we can rewrite (B.16) in the equivalent form: 


$$
1-2 \frac{D^{\prime(W)}(1)}{\left(R_{W, W}(1)-1\right) D^{(W-1)}(1)} \varepsilon \cos \varphi+o(\varepsilon)<1
$$

The validity of (B.18) immediately follows from the below inequalities:

$$
\begin{gathered}
\frac{D^{(W)}(1)}{D^{(W-1)}(1)}<0 \quad(\text { see Theorem } 1) \\
R_{W, W}(1)-1<0, \quad \cos \varphi>0 .
\end{gathered}
$$

Thus we verified inequality (B.14).

This latter, the result of the Corollary B.2, and Rouche's theorem yield that the determinant $D^{(W)}(z)$ has exactly $W+1$ zeros in the region $S_{\varepsilon}, \varepsilon \rightarrow 0$.

\section{Appendix C}

Coefficients $\psi_{v}^{(l)}, l \geq 0, v=\overline{0, W}$, are defined by the formulas:

$$
\psi_{v}^{(0)}=\widetilde{\psi}_{v}^{(0)}+\widetilde{\psi}_{v}^{(-1)}, \psi_{v}^{(l)}=\tilde{\psi}_{v}^{(l)}, l \geq 1
$$

where values $\tilde{\psi}_{v}^{(l)}, l \geq-1$, are defined as follows:

1. Variant 1.a:

$$
\tilde{\psi}_{v}^{(l)}=\sum_{m=0}^{W} q_{m}\left[\sum_{k=0}^{\min \{v, W-m\}} \sum_{r=0}^{k+m} \theta_{v, k, k+m, r}^{(l+1)}+\sum_{k=W-m+1}^{W} \chi_{1, v, k}^{(l+1)}\right]
$$

2. Variant 1.b:

$$
\tilde{\psi}_{v}^{(l)}=\sum_{m=0}^{W} q_{m}\left[\sum_{k=0}^{W-m} \sum_{r=m}^{W} \theta_{v, k, k, r-m}^{(l+1)}+\sum_{k=W-m+1}^{W} \chi_{1, v, k}^{(l+1)}\right]
$$

3. Variant 2.a:

$\tilde{\psi}_{v}^{(l)}$

$$
=\sum_{m=0}^{W} q_{m}\left[\sum_{i=0}^{l+1} \sum_{r=0}^{W} \chi_{2, W, r}^{(l+1-i)} \sum_{k=W+1-m}^{W} \sum_{t=0}^{i} \chi_{1, v, k}^{(i-t)} v_{t}(m, k)+\sum_{k=0}^{\min \{v, W-m\}} \sum_{r=0}^{k+m} \theta_{v, k, k+m, r}^{(l+1)}\right],
$$

4. Variant 2.b:

$$
\begin{aligned}
& \widetilde{\psi}_{v}^{(l)} \\
& =\sum_{m=0}^{W} q_{m}\left[\sum_{k=0}^{W-m} \sum_{r=m}^{W} \theta_{v, k, k, r-m}^{(l+1)}+\sum_{k=W-m+1}^{W} \sum_{i=0}^{l+1} \sum_{t=0}^{i} \chi_{1, v, k}^{(i-t)} v_{t}(m, k) \sum_{r=m}^{W} \chi_{2, W-m, r-m}^{(l+1-i)}\right],
\end{aligned}
$$

where 


$$
\begin{gathered}
\chi_{n, v, r}^{(k)}=\left\{\begin{array}{c}
\frac{(-\lambda)^{k}}{k !} \int_{0}^{\infty} t^{k} \delta_{v, r}(t) d B_{n}(t), v \geq r, \\
0, v<r, n=1,2,
\end{array}\right. \\
\sum_{r=0}^{v} \chi_{n, v, r}^{(0)}=1, \sum_{r=0}^{v} \chi_{n, v, r}^{(1)}=-\lambda b_{1}^{(n)}, \sum_{r=0}^{v} \chi_{n, v, r}^{(2)}=\frac{\lambda^{2} b_{2}^{(n)}}{2}, \\
v_{n}(m, k)=\left\{\begin{array}{c}
\theta_{v, k, k+m, r}^{(h)} \sum_{l=0}^{h} \chi_{1, v, k}^{(l)} \chi_{2, k+m, r}^{(h-l)}, \quad h \geq 0, \\
-\left(\Gamma_{3}(m, k)+3 \Gamma_{1}(m, k) \Gamma_{2}(m, k)+\Gamma_{1}^{3}(m, k)\right) / 6, n=3, \quad n=1, \\
\left(\Gamma_{2}(m, k)+\left(\Gamma_{1}(m, k)\right)^{2}\right) / 2, n=2, \\
\Gamma_{r}(m, k)=\left(\frac{\lambda}{\gamma}\right)_{n=W}^{r} \sum_{W-m+1}^{k} \frac{1}{n^{r}}, \quad r \geq 1 .
\end{array}\right.
\end{gathered}
$$

Coefficients $\Xi_{v}^{(l)}$ in formula (17) are defined as follows:

$$
\begin{gathered}
\Xi_{v}^{(0)}=\psi_{v}^{(0)}-\sum_{m=0}^{W} \nabla_{v, m} \psi_{m}^{(0)}+1 \\
\Xi_{v}^{(1)}=\psi_{v}^{(1)}+\sum_{m=0}^{W} \nabla_{v, m} \psi_{m}^{(0)}-\sum_{m=0}^{w} \nabla_{v, m} \psi_{m}^{(1)}-1 \\
\Xi_{v}^{(l)}=\psi_{v}^{(l)}+\sum_{m=0}^{W} \nabla_{v, m}\left(\psi_{m}^{(l-1)}-\psi_{m}^{(l)}\right), l \geq 2 .
\end{gathered}
$$




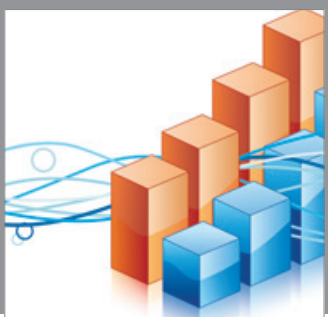

Advances in

Operations Research

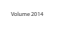

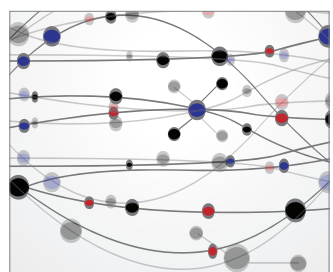

\section{The Scientific} World Journal
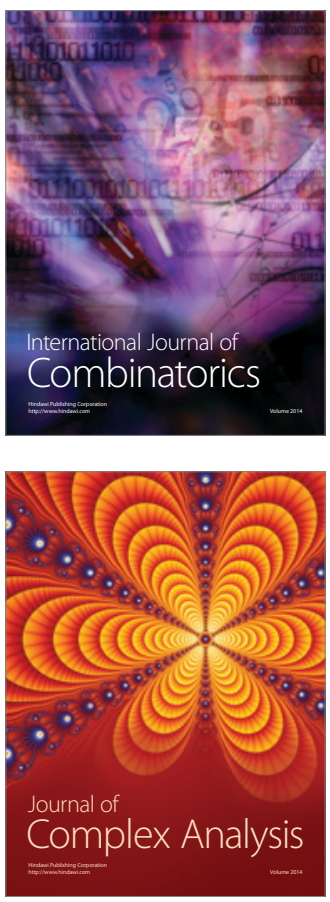

International Journal of

Mathematics and

Mathematical

Sciences
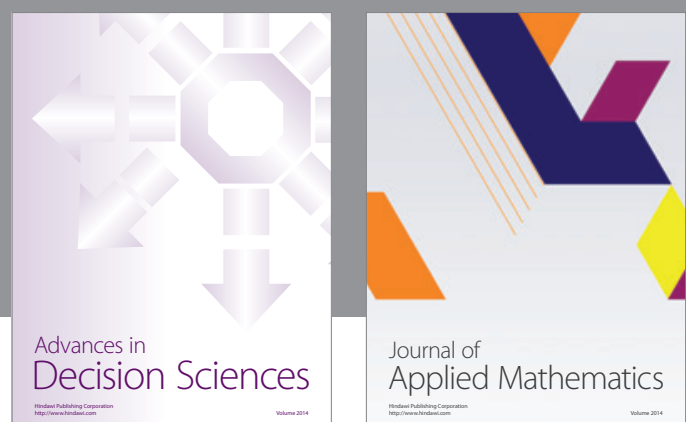

Journal of

Applied Mathematics
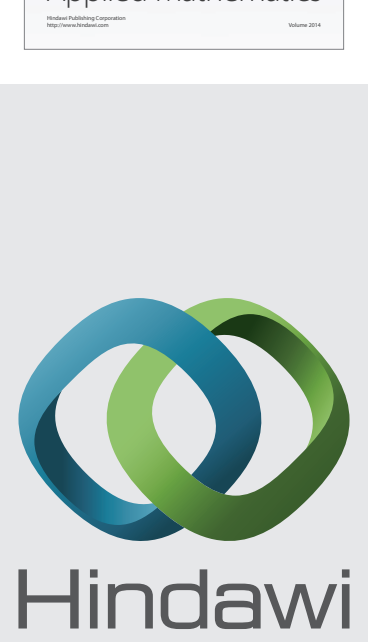

Submit your manuscripts at http://www.hindawi.com
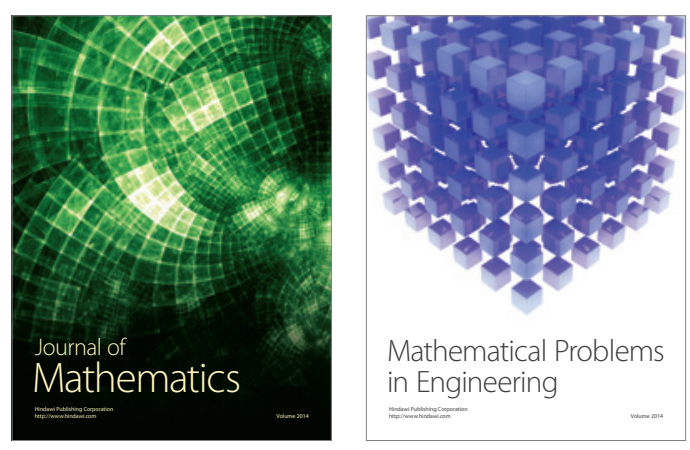

Mathematical Problems in Engineering
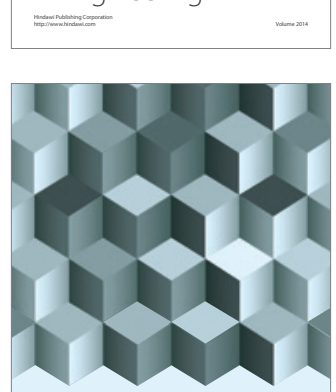

Journal of

Function Spaces
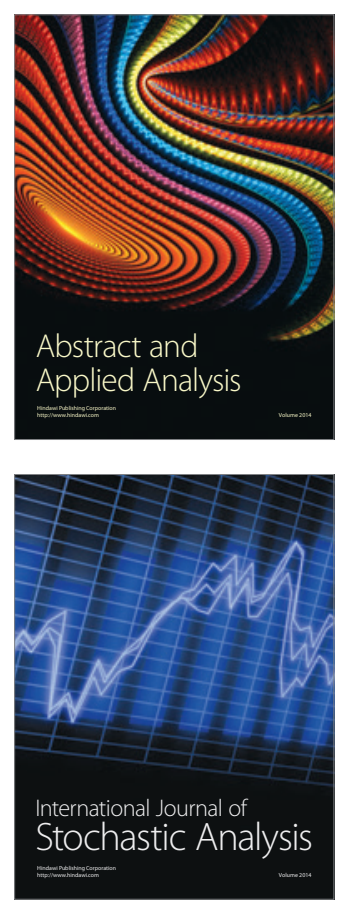

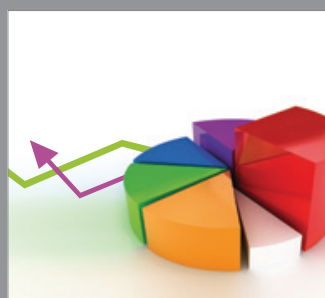

ournal of

Probability and Statistics

Promensencen
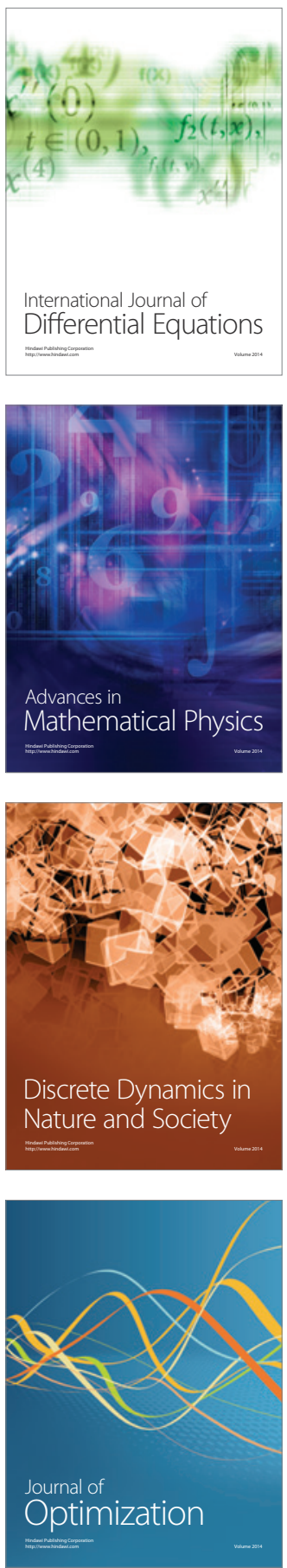